quels résultats nos expériences devraient donner. Bref, nous sommes dans une situation assez semblable à l'abbé Gregor Mendel, qui, lui aussi, a dû avoir une foi inébranlable en sa propre théorie. Nous sommes donc souvent tentés d'élaguer des résultats qui ne nous conviennent pas. Cette situation $m$ 'inquiète, même si je crois que nous sommes mûs par un esprit honnête. Non, mon inquiétude vient de ce que notre comportement s'inscrit en porte-à-faux par rapport au mode de travail idéalisé que le grand public prête aux scientifiques. Nous sommes à mille lieues d'une recherche impersonnelle, objective, et dénuée de passion.

Ces lectures ont réellement semé un doute dans mon esprit. J'ai trouvé quelque réconfort chez Albert Einstein, qui a écrit, dans une lettre à Heisenberg: «C'est la théoriequi décide c'e que nous pouvons observer». C'est bien ce que Mendel a fait, et c'est bien ce que nous faisons tous les jours.

Si Einstein ne constitue pas une référence suffisante, voici un passage de Sigmund Freud que mon épouse m'a montré hier [2]: "Wir haben oftmals die Forderung vertreten gehört, dass eine Wissenschaft über klaren und scharf definierten Grundbegriffen aufgehaut sein soll. In Wirklichkeit beginnt keine Wissenschaft mit solchen Definitionen, auch die exakteste nicht. Der richtige Anfang der wissenschaftlichen Tätigkeit besteht vielmehr in der Beschreibung von Erscheinungen, die dann weiterhin gruppiert. angeordnet und in Zusammenhänge eingetragen werden. Schon bei der Beschreibung kann man es nicht vermeiden, gewisse ab- strakte Ideen auf das Material anzuw'enden, die man irgendwoher, gewiss nicht aus der neuen Erfahrung allein, herbeiholt.»

Vous voyez que Freud et Einstein tiennent essentiellement le même langage. Vous voyez aussi qu'il y a une tension, pour ne pas dire une contradiction, sur des questions très fondamentales. Qu'est-ce que tout cela peut bien avoir à faire à nos travaux? Et bien, je soutiens que c'est dans cette tension que réside tout l'intérêt de la recherche, et qu'elle prend des formes particulièrement fécondes dans le domaine de la chimie moderne.

Pour finir, quelques mots à propos du prix Latsis. Vous savez que ce prix n'est pas purement honorifique, encore que, jusqu'à présent, seuls quelques journalistes en quête de sensations fortes ont voulu savoir ce que nous entendions faire d'une si belle somme. Eh bien, sachez que jusqu'ici, nous nous sommes bornés à acheter l'œuvre complète de Freud, dont vous venez d'entendre un extrait. D'autres folies suivront! Mais audelà de ces petits fantasmes, il y a surtout le sens d'une grande liberté. La liberté de rêver, mais aussi celle de s'engager dans certains débats. Fortifié d'une autorité nouvelle, je me sens en droit d'exprimer une opinion sur certains sujets d'actualité. En politique de la science, certes, mais au-delà, je crois qu'il faut s'engager sur des grands débats comme celui de l'énergie nucléaire. Non nécessairement pour plaider qu'on y renonce purement et simplement, mais surtout pour apporter une touche modératrice dans un débat par trop passionné. Pour éviter que l'on peigne le diable sur la muraille, mais aussi pour modérer les partisans qui font souvent preuve d'une confiance trop naïve en la technologie. J'ai été souvent tenté de m'engager dans ce débat (par exemple en adressant une lettre de lecteur à un grand quotidien), maisj'ai toujours craint de n'être pas pris au sérieux. Eh bien, aujourd hui je crois que quelques-uns au moins m'écouteront. C'est surtout de cette force nouvelle que je suis reconnaissant. Reconnaissant au Conseil du Fonds national, et reconnaissant à la Fondation Latsis.

Monsieur le Conseiller fédéral, Mesdames et Messieurs, cher amis, je vous remercie de votre attention.

Reçu le 14 décémbre 1990

[1] W. Broad, N. Wade, "Betrayers of the truth". Simon and Schuster, New York, 1982, "La souris truquée», trad. C. Jeanmougin. Seuil, Paris, 1987.

[2] S. Freud, "Triebe und Tricbschicksale", 1915 (Studienausgabe S. Fischer Verlag. Frankfurt. Band III, S. 81). Voici la traduction française par J. Laplanche et J.-B. Pontalis (dans «Mctapsychologie», Gallimard, Paris, p. 11): «Nous an'oms son'ent entendu formuler levigence smirante: whe scionce doit être construite sur des's come'pts fondamentaux chairs et netteme'nt difinis. En réalitó, ancume science. mômo la plus cisacte., ne conmence par de lelles définitions. Le víritable'

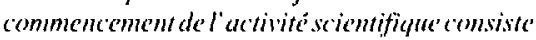
plurôr dans la description de phéneminess, yui

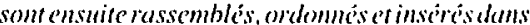
des relations. Dans la description. dijai, an ne peat éviter d'appliquer all materiel iertaines idées abstraites que' l'on puise ici ou lì c't erertuinement pas dans la sealc expériene' acturelle',n

Chimia 45 (1991) 193-195

(C) Schweiz. Chemiker-Verband: ISSN 0009-4293

\title{
Der neue Entwurf des Schweizerischen Umweltschutzgesetzes
}

\author{
Georg Karlaganis*
}

\section{Die schweizerische Umweltschutzge- setzgebung}

Ausser dem Umweltschutzgesetz(USG) befassen sich noch zahlreiche andere Bundesgesetze mit den Anliegen des Umweltschutzes. Zur Zeit der Gründung der Berner

*Korrespondenz: PD Dr. Georg Karlaganis Bundesamt für Umwelt, Wald und Landschaft Hallwylstrasse 4 $\mathrm{CH}-3003$ Bern
Chemischen Gesellschaft vor 100 Jahren existierte allerdings erst das Fabrikgesetz/ Arbeitsgesetz (1877/1964). Es folgten das Forstpolizeigesetz (1902), das Gewässerschutzgesetz (1955/1971), das Natur- und Heimatschutzgesetz (1966), das Fischereigesetz (1973) und das Raumplanungsgesetz (1979).

Im Jahre 1971 ist unsere Bundesverfassung mit einem Umweltschutz-Artikel (Art. $24^{\text {scpics }}$ ) ergänzt worden. Dieser Verfassungsauftrag führte zu unserem Unweltschutzgesetz, welches seit dem 1. Januar 1985 in Kraft ist. Der vom Verfassungsartikel anvi- sierte Schutz gilt dem Menschen und seiner natürlichen Umwelt. Schutzobjekt sind neben der menschlichen Gesundheit, welche das psychische Wohlbefinden einschliesst, auch die Umweltelemente selbst als Grundlage allen Lebens.

Der Zweckartikel des Umweltschutzgesetzes gibt dem wie folgt Ausdruck: «Dieses Gesetz soll Menschen, Tiere und Pflanzen, ihre Lebensgemeinschaften und Lebensräume gegen schädliche oder lästige Einwirkungen schützen und die Fruchtbarkcit des Bodens erhalten» (Art. 1, Absatz 1).

Das Umweltschutzgesetz basiert auf mehreren wichtigen Grundprinzipien: dem Vorsorgeprinzip, dem Prinzip der Bekämpfung an der Quelle, dem Verursacherprinzip, dem Kooperationsprinzip und dem Prinzip der ganzheitlichen Betrachtungsweise.

Das Umweltschutzgesetz regelt die Bereiche Luftreinhaltung, Lärmbekämpfung, Abfälle, umweltgefährdende Stoffe und Schutz des Bodens. Das Unweltschutzgesetz ist ein Rahmengesetz. Die Konkretisierung erfolgte durch eine Reihe von Verordnungen: Luftreinhalte-Verordnung (LRV, 1986), Lärmschutz-Vcrordnung (LSV. 
quels résultats nos expériences devraient donner. Bref, nous sommes dans une situation assez semblable à l'abbé Gregor Mendel, qui, lui aussi, a dû avoir une foi inébranlable en sa propre théorie. Nous sommes donc souvent tentés d'élaguer des résultats qui ne nous conviennent pas. Cette situation $m$ 'inquiète, même si je crois que nous sommes mûs par un esprit honnête. Non, mon inquiétude vient de ce que notre comportement s'inscrit en porte-à-faux par rapport au mode de travail idéalisé que le grand public prête aux scientifiques. Nous sommes à mille lieues d'une recherche impersonnelle, objective, et dénuée de passion.

Ces lectures ont réellement semé un doute dans mon esprit. J'ai trouvé quelque réconfort chez Albert Einstein, qui a écrit, dans une lettre à Heisenberg: «C'est la théoriequi décide c'e que nous pouvons observer». C'est bien ce que Mendel a fait, et c'est bien ce que nous faisons tous les jours.

Si Einstein ne constitue pas une référence suffisante, voici un passage de Sigmund Freud que mon épouse m'a montré hier [2]: "Wir haben oftmals die Forderung vertreten gehört, dass eine Wissenschaft über klaren und scharf definierten Grundbegriffen aufgehaut sein soll. In Wirklichkeit beginnt keine Wissenschaft mit solchen Definitionen, auch die exakteste nicht. Der richtige Anfang der wissenschaftlichen Tätigkeit besteht vielmehr in der Beschreibung von Erscheinungen, die dann weiterhin gruppiert. angeordnet und in Zusammenhänge eingetragen werden. Schon bei der Beschreibung kann man es nicht vermeiden, gewisse ab- strakte Ideen auf das Material anzuw'enden, die man irgendwoher, gewiss nicht aus der neuen Erfahrung allein, herbeiholt.»

Vous voyez que Freud et Einstein tiennent essentiellement le même langage. Vous voyez aussi qu'il y a une tension, pour ne pas dire une contradiction, sur des questions très fondamentales. Qu'est-ce que tout cela peut bien avoir à faire à nos travaux? Et bien, je soutiens que c'est dans cette tension que réside tout l'intérêt de la recherche, et qu'elle prend des formes particulièrement fécondes dans le domaine de la chimie moderne.

Pour finir, quelques mots à propos du prix Latsis. Vous savez que ce prix n'est pas purement honorifique, encore que, jusqu'à présent, seuls quelques journalistes en quête de sensations fortes ont voulu savoir ce que nous entendions faire d'une si belle somme. Eh bien, sachez que jusqu'ici, nous nous sommes bornés à acheter l'œuvre complète de Freud, dont vous venez d'entendre un extrait. D'autres folies suivront! Mais audelà de ces petits fantasmes, il y a surtout le sens d'une grande liberté. La liberté de rêver, mais aussi celle de s'engager dans certains débats. Fortifié d'une autorité nouvelle, je me sens en droit d'exprimer une opinion sur certains sujets d'actualité. En politique de la science, certes, mais au-delà, je crois qu'il faut s'engager sur des grands débats comme celui de l'énergie nucléaire. Non nécessairement pour plaider qu'on y renonce purement et simplement, mais surtout pour apporter une touche modératrice dans un débat par trop passionné. Pour éviter que l'on peigne le diable sur la muraille, mais aussi pour modérer les partisans qui font souvent preuve d'une confiance trop naïve en la technologie. J'ai été souvent tenté de m'engager dans ce débat (par exemple en adressant une lettre de lecteur à un grand quotidien), maisj'ai toujours craint de n'être pas pris au sérieux. Eh bien, aujourd hui je crois que quelques-uns au moins m'écouteront. C'est surtout de cette force nouvelle que je suis reconnaissant. Reconnaissant au Conseil du Fonds national, et reconnaissant à la Fondation Latsis.

Monsieur le Conseiller fédéral, Mesdames et Messieurs, cher amis, je vous remercie de votre attention.

Reçu le 14 décémbre 1990

[1] W. Broad, N. Wade, "Betrayers of the truth". Simon and Schuster, New York, 1982, "La souris truquée», trad. C. Jeanmougin. Seuil, Paris, 1987.

[2] S. Freud, "Triebe und Tricbschicksale", 1915 (Studienausgabe S. Fischer Verlag. Frankfurt. Band III, S. 81). Voici la traduction française par J. Laplanche et J.-B. Pontalis (dans «Mctapsychologie», Gallimard, Paris, p. 11): «Nous an'oms son'ent entendu formuler levigence smirante: whe scionce doit être construite sur des's come'pts fondamentaux chairs et netteme'nt difinis. En réalitó, ancume science. mômo la plus cisacte., ne conmence par de lelles définitions. Le víritable'

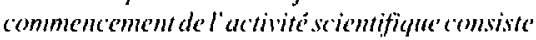
plurôr dans la description de phéneminess, yui

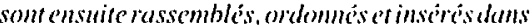
des relations. Dans la description. dijai, an ne peat éviter d'appliquer all materiel iertaines idées abstraites que' l'on puise ici ou lì c't erertuinement pas dans la sealc expériene' acturelle',n

Chimia 45 (1991) 193-195

(C) Schweiz. Chemiker-Verband: ISSN 0009-4293

\title{
Der neue Entwurf des Schweizerischen Umweltschutzgesetzes
}

\author{
Georg Karlaganis*
}

\section{Die schweizerische Umweltschutzge- setzgebung}

Ausser dem Umweltschutzgesetz(USG) befassen sich noch zahlreiche andere Bundesgesetze mit den Anliegen des Umweltschutzes. Zur Zeit der Gründung der Berner

*Korrespondenz: PD Dr. Georg Karlaganis Bundesamt für Umwelt, Wald und Landschaft Hallwylstrasse 4 $\mathrm{CH}-3003$ Bern
Chemischen Gesellschaft vor 100 Jahren existierte allerdings erst das Fabrikgesetz/ Arbeitsgesetz (1877/1964). Es folgten das Forstpolizeigesetz (1902), das Gewässerschutzgesetz (1955/1971), das Natur- und Heimatschutzgesetz (1966), das Fischereigesetz (1973) und das Raumplanungsgesetz (1979).

Im Jahre 1971 ist unsere Bundesverfassung mit einem Umweltschutz-Artikel (Art. $24^{\text {scpics }}$ ) ergänzt worden. Dieser Verfassungsauftrag führte zu unserem Unweltschutzgesetz, welches seit dem 1. Januar 1985 in Kraft ist. Der vom Verfassungsartikel anvi- sierte Schutz gilt dem Menschen und seiner natürlichen Umwelt. Schutzobjekt sind neben der menschlichen Gesundheit, welche das psychische Wohlbefinden einschliesst, auch die Umweltelemente selbst als Grundlage allen Lebens.

Der Zweckartikel des Umweltschutzgesetzes gibt dem wie folgt Ausdruck: «Dieses Gesetz soll Menschen, Tiere und Pflanzen, ihre Lebensgemeinschaften und Lebensräume gegen schädliche oder lästige Einwirkungen schützen und die Fruchtbarkcit des Bodens erhalten» (Art. 1, Absatz 1).

Das Umweltschutzgesetz basiert auf mehreren wichtigen Grundprinzipien: dem Vorsorgeprinzip, dem Prinzip der Bekämpfung an der Quelle, dem Verursacherprinzip, dem Kooperationsprinzip und dem Prinzip der ganzheitlichen Betrachtungsweise.

Das Umweltschutzgesetz regelt die Bereiche Luftreinhaltung, Lärmbekämpfung, Abfälle, umweltgefährdende Stoffe und Schutz des Bodens. Das Unweltschutzgesetz ist ein Rahmengesetz. Die Konkretisierung erfolgte durch eine Reihe von Verordnungen: Luftreinhalte-Verordnung (LRV, 1986), Lärmschutz-Vcrordnung (LSV. 
1987), Verordnung über umweltgefährdende Stoffe (StoV, 1986), Verordnung über Schadstoffe im Boden (VSBo, 1986), Verordnung über den Verkehr mit Sonderabfällen (VVS, 1987), Technische Verordnung über Abfälle (TVA, 1991), Verordnung über die Umweltverträglichkeitsprüfung (UVPV, 1989), Störfall-Verordnung (StFV, 1991). Die Instrumente sind polizeirechtliche Instrumente: Emissionsgrenzwerte, Bau- und Ausrïstungsvorschriften, Verkehrs- oder Betriebsvorschriften und ähnliches. Seit 1985 konzentrierten sich Bund, Kantone und Gemeinden hauptsächlich auf den Vollzug des USG. Die Erfahrungen aus dem Vollzug einerseits und die rasche technologische Entwicklung unserer Gesellschaft andererseits zeigten, dass es zweckmässig ist, neue Regelungsbereiche in das Gesetz einzugliedern.

Am 16. Mai 1990 hat der schweizerische Bundesrat das Eidgenössische Departement des Innern ermächtigt, über eine Änderung des Umweltschutzgesetzes bei Kantonen, Wirtschafts- und Fachorganisationen eine Vernehmlassung durchzuführen. Diese Vernehmlassung hat im Jahre 1990 stattgefunden. DerEntwurf umfasste die folgenden vier voneinander unabhängigen Bereiche:

- Regelung des Umgangs mit umweltgefährdenden Organismen;

- Ergänzung der Vorschriften über Abfälle;

- Erhebung von Lenkungsabgaben auf bestimmte umweltgefährdende Stoffe und Erzeugnisse;

- Förderung der Entwicklung von Umweltschutztechnologien.

\section{Die Lücken im Bereich der Biotechno- logie}

Eingehende Abklärungen der rechtlichen Lage haben gezeigt, dass die bestehenden schweizerischen Gesetze die Umweltaspekte im Bereich der Biotechnologie nur ungenügend abdecken. Zwar können Sicherheitsfragen bei der Anwendung von Mikroorganismen in geschlossenen Systemen über das heutige Umweltschutzgesetz (Art. 10) geregelt werden, und ein entsprechender Ausführungserlass (Störfallverordnung) wurde am 27. Februar 1991 vom Bundesrat beschlossen.

Im USG bestehen hingegen Lücken in folgenden Bereichen:

- Freisetzung von Organismen zu Versuchszwecken (z.B. Prüfung der Wirksamkeit und der Nebenwirkungen);

- Anwendung von Organismen in der Umwelt (Rohstoffabbau, Abfallentsorgung, Schädlingsbekämpfung);

- Einfuhr und Handel mit Zierpflanzen (Quarantäne zum Schutz von besonders gefährlichen Schädlingen, Krankheiten oder Unkräutern).
Mit der vorgeschlagenen Neuregelung will der Bundesrat drei Ziele erreichen:

1) Die erkannten gesetzlichen Lücken sollen geschlossen werden. Eine klare gesetzliche Grundlage soll dafür sorgen, dass im Umgang mit Organismen die Umweltaspekte ausreichend berücksichtigt werden.

2) Eine verantwortungsbewusste Forschung, Entwicklung und Anwendung der Bio- und Gentechnologie soll auch künftig möglich bleiben. Die neue Regelung soll somit kein generelles Verbot oder Moratorium von Forschung, Entwicklung und Anwendung im Bereich der Bio- und Gentechnologie sein.

3) Die neue Regelung soll mit den entsprechenden europäischen Regelungen harmonisiert sein. Im Bereich der Gentechnologie soll eine Rechtsgrundlage geschaffen werden, die den beiden kürzlich von der EG erlassenen Richtlinien über die Anwendung genetisch verwendeter Mikroorganismen in geschlossenen Systemen beziehungsweise in der Umwelt entspricht. Im Bereich der Quarantäne-Organismen will die Schweiz die Verpflichtungen im Rahmen des internationalen Abkommens über Pflanzenschutz erfüllen.

Die Regelung der neuen Technologien im Bereich der Umwelt soll in die bestehende Umweltschutzgesetzgebung eingebettet und nicht in einem neuen Spezialgesetz geregelt werden. Sie soll im weiteren auch nicht auf genetisch veränderte Organismen beschränkt sein, sondern alle Organismen, welche die Umwelt gefährden können, gleichermassen erfassen und deshalb sowoh natürliche und gezüchtete, als auch genetisch veränderte Organismen betreffen. Als Instrument dieser Regelung sind einerseits Vorsorgemassnahmen wie Melde- und Bewilligungsverfahren sowie die Möglichkeit spezifischer Verbote, anderseits aber auch Abwehr- und Bekämpfungsmassnahmen vorgesehen.

Kernstück der Vorlage im Bereich der Biotechnologie sind drei neue Artikel (Art. 29 a-c), die als neues Kapitel das Umweltschutzgesetz ergänzen sollen. Die vorgeschlagenen neuen Art. 29 a und 29 b betreffen die Herstellung und Einfuhr, das Inverkehrbringen und die Anwendung umweltgefährdender Organismen. Analog zu den Art. 26-28 des Umweltschutzgesetzes für den Stoffbereich beruhen auch sie auf der Eigenverantwortung der Handelnden. Die Aufgaben der Behörden werden in Art. $29 \mathrm{c}$ (analog zu Art. 29 im Chemikalienbereich) aufgelistet.

Wie bereits die Regelung der umweltgefährdenden Stoffe im Umweltschutzgesetz sieht auch der Entwurf einer Regelung über umweltgefährdende Organismen mehrheitlich Bundesvollzug vor, Dabei sollen bestehende Strukturen und eingespielte Verfahren soweit wie möglich berücksichtigt werden.

CHIMIA 45 (1991) Nr. 6 (Juni)

3. Ergänzung der Vorschriften über Abfälle

Im Bereich Abfälle bestehen heute drei grosse Schwierigkeiten:

I) Die Kapazität der zur Verfügung stehenden Anlagen reicht nicht aus, um die parallel zur Wirtschaftsentwicklung angestiegene Abfallmenge $\mathrm{zu}$ behandeln und/oder zu entsorgen.

2) Es entsprechen noch nicht alle Anlagen dem Stand der Technik und den geltenden Vorschriften.

3) Möglichkeiten der Abfallvermeidung und Abfallverwertung sind nicht ausgeschöpft.

Die mangelnde Kapazität der Anlagen bereitet grosse Schwierigkeiten im Bereich der Sonderabfälle. Beträchtliche Mengen von Sonderabfällen müssen heute wegen fehlender eigener Anlagen ins europäische Ausland exportiert werden.

Technisch hochstehende Abfallbehandlungsanlagen sind teuer. Die Investitionen für eine Verbrennungsanlage für Sonderabfälle gehen in die Grössenordnung von 100 Mio. Fr. Eine Anlage zur Aufarbeitung von Batterien kostet schätzungsweise 10 bis 30 Mio. Fr. Dies sind, volkswirtschaftlich gesehen, zwar recht geringe Beträge, übersteigen aber die finanziellen Möglichkeiten der heutigen, in eher kleineren Betrieben strukturierten Abfallwirtschaft.

Die grundsätzliche Verantwortung der Inhaber von Abfällen zur umweltgerech-ten Entsorgung ist zwar verankert. In der Praxis sind die Betroffenen aber in der Regel nicht in der Lage, die nötigen Anlagen selber $\mathrm{zu}$ bauen und $\mathrm{zu}$ betreiben. Die zentralen Elemente der Vorlage sind die Sicherung der Finanzierung der Entsorgung durch die vorgezogene Entsorgungsgebühr und die Bildung von Entsorgungsregionen. Damit soll der Bundesrat die Möglichkeit erhalten, tätig zu werden, wenn die Abfallwirtschaft und die Kantone organisatorisch und finanziell nicht in der Lage sind, diese Einrichtungen zu realisieren. Im weiteren erhielte der Bundesrat die Kompetenz, den Handel mit ausländischen Sonderabfällen durch Firmen mit Sitz in der Schweiz zu kontrollieren.

\section{Lenkungsabgaben}

In den vergangenen Jahren ist von verschiedenen Seiten der Ruf laut geworden, den umweltpolitischen Werkzeugkasten mit ökonomischen Instrumenten zu ergänzen. $\mathrm{Zu}$ den ökonomischen Instrumenten gehören auch die Lenkungsabgaben. Der Einsatz von Lenkungsabgaben wird vom Bundesrat in drei Bereichen vorgeschlagen:

- Lenkungsabgabe auf flüchtigen organischen Kohlenwasserstoffen;

- Lenkungsabgabe auf Heizöl «extra 
leicht» und Dieselöl mit einem Schwefelgehalt von mehr als $0,1 \%$ (\%-Masse);

- Lenkungsabgabe auf Handelsdüngern und Pflanzenbehandlungsmitteln.

Mit solchen Lenkungsabgaben soll ein marktwirtschaftliches Instrument in die schweizerische Umweltpolitik eingebaut werden. Von der Lenkungsabgabe auf flüchtigen organischen Kohlenwasserstoffen (VOC) werden vorwiegend Lösungsmittel, Treibgase oder Kühlmittel erfasst. Die Abgabe beträgt 2 bis $3 \mathrm{Fr} . / \mathrm{kg}$ VOC. Die Lenkungsabgabe auf Heizöl "extra leicht» soll die Luftbelastung durch Schwefel weiter reduzieren. Erfasst würden aber nurHeizöl «extra leicht» mit einem Schwefelgehalt von mehr als 0,1 Massen-\%. Hier wird an eine Abgabe in der Grössenordnung zwischen Fr. 1.50 und Fr. 1.80 pro $100 \mathrm{~kg}$ gedacht. Die Abgabe auf Handelsdüngern und Pflanzenbehandlungsmitteln trifft in erster Linie die Landwirtschaft und soll zur Förderung einer weniger intensiven Agrarproduktion beitragen. Die anvisierten Produkte würden zwischen 30 und $50 \%$ verteuert. Diese Lenkungsabgaben würden Einnahmen in der Grössenordnung von 450-500 Mio. Fr. pro Jahr bringen.
Zusätzlich zu diesen Lenkungsabgaben hat der Bundesrat 1990 auch noch den Auftrag erteilt, eine Vorlage für eine $\mathrm{CO}_{2}-\mathrm{Ab}$ gabe zur Verbrauchsreduktion der fossilen Brenn- und Treibstoffe vorzubereiten. Dieses Instrument soll allerdings nicht im USG sondern in einer eigenen gesetzlichen Grundlage verankert werden.

\section{Förderung der Entwicklung von Um- weltschutztechnologien}

Die Vorlage bezweckt, die Entwicklung neuer Umweltschutztechnologien durch den Bund zu fördern. Dem Bund soll ein Instrument zur Verfügung gestellt werden, das ihm erlaubt, die Umsetzung wissenschaftlicher Erkenntnisse in der Praxis zu unterstüzen. Gedacht wird an Beiträge oder Subventionen an die Entwicklungskosten verbesserter Anlagen und Verfahren, die zur Reduktion der Umweltbelastung beitragen.

Besonders gross ist der Bedarf nach neuen und verbesserten Recyling-Technologien und nach produktionsintegrierten Umweltschutztechnologien, welche die Entstehung von Emissionen oder Abfällen vermindern oder ganz vermeiden.

\section{Ausblick}

Mit der USG-Änderung, wie sie in die Vernehmlassung gegeben worden ist, wird ein weiterer wichtiger umweltpolitischer Schritt getan. Er hilft mit, noch bestehende Lücken zu schliessen und entspricht dem fortschrittlichen Bild, das die Schweiz in internationaler Hinsicht im Bereich der Umweltpolitik bietet. Die Resultate der Vernehmlassung werden zur Zeit ausgewertet.

Eingegangen am 14. März 1991

[1] Umweltschutzgesetz (USG) vom 7. Oktober 1983; in Kraft seit I. Januar 1985.

[2] Erläuterungen zum Umweltschutzgesetz (BUWAL August 1988)

[3] Änderung des Umweltschutzgesetzes (USG). Entwurf und erläuternder Bericht. Eidgenössisches Departement des Innern. Mai 1990

[4] A. Mohr, USG-Änderung bringt neue Massnahmen (BUWAL-Bulletin 3/90).

[5] H. Hosbach, Biotechnologie-Regelung im neuen Entwurf des Schweizerischen Umweltschutzgesetzes. Umweltwissenschaften und Schadstoffforschung, Z. Umuellchiem. Ökotoxikol. 1990, 2, $142-143$.

[6] Pressemitteilung $\mathrm{CO}_{2}$-Abgabe.

\section{Einführung}

Enantiomerenreines ( $1 S, 4 R)-4-\mathrm{Hy}$ droxycyclopent-2-enyl-acetate (1) gehört zu einer Klasse optisch aktiver CyclopentenDerivate, die als Bausteine für die Synthese von Prostaglandinen [1-4] und anderen cyclopentanoiden Naturstoffen [5] eingesetzt werden können.

Die enzymkatalysierte Synthese des Cyclopentenderivats 1 durch enantioselektive Hydrolyse der entsprechenden cisDiester 2 wurde bereits mehrmals beschrieben [2][6-9]. Die Konfiguration des entstehenden chiralen Monoesters ist im hohen Masse vom eingesetzten Biokatalysator abhängig. Die meisten Esterasen [7-9], Chymotrypsin [7] und Bäckerhefe [1][6][7] begünstigen die Spaltung der Re-Estergruppe des meso-Diesters 2 und somit die Bildung des ( $1 S, 4 R)$-Monoesters 1. Lipasen dagegen katalysieren die asymmetrische Hydrolyse des cis-Diesters 2 mit umgekehrter Enantioselektivität zum Monoester

Alexander Knoll'), Monika Böhme'), Steffen Merker'), Gerd Fabian'), Barbara Häfner') und Fritz Theil²)

Abstract. $(1 S, 4 R)-4-H y d r o x y c y c l o p e n t-2$-enyl-acetate (1), an attractive starting material for the synthesis of prostaglandins, was readily prepared by an enzyme-catalyzed interesterification procedure using acetic anhydride as acylation agent. As the chemical yield of the chiral monoacylation product is rather low $(45 \%)$, we investigated the acylation mechanism of this reaction to optimize the product output. Kinetic measurements were carried out by means of gas chromatography on a chiral stationary phase, synthesized by methylation of $\beta$-cyclodextrin. ent-1 [7][10][12] (Schema 1).

Wird Schweinepankreaslipase (in Form des Rohpräparates Pankreatin) in wasserfreien Medien für die Veresterung des mesoDiols 3 eingesetzt, wird die Si-OH-Gruppe acyliert [13]. Auf diese Weise ist das in

*Korrespondenz: Dr. A. Knoll')

1) FZB Biotechnik $G m b H$, Alt-Stralau 62 D-1017 Berlin

2) Zentralinstitut für Organische Chemie Rudower Chaussee 5, D-1 199 Berlin 